\title{
Lumbar Drain Tunneling: A Safe Two-Needle Technique
}

\author{
Srinivas Deepti ${ }^{1}$ Halesh Ashwini ${ }^{2} \quad$ Keshavan Venkatesh ${ }^{1}$ \\ ${ }^{1}$ Division of Neuroanaesthesia and Critical Care, Apollo Hospitals, \\ Bannerghatta Road, Bengaluru, Karnataka, India \\ ${ }^{2}$ Division of Neuroanaesthesia and Critical Care, Sagar Hospitals, \\ Bengaluru, Karnataka, India

\begin{abstract}
Address for correspondence Srinivas Deepti, MD, Division of Neuroanaesthesia and Critical Care, Apollo Hospitals, Bannerghatta Road, Bengaluru 560076, Karnataka, India (e-mail: bsdeepti@gmail.com).
\end{abstract}

J Neuroanaesthesiol Crit Care:2021;8:69-70
Abstract
Keywords
- lumbar drain
- lumbar drain
tunneling
- Tuohy needle

Lumbar drain catheter insertion for prolonged periods of time is necessary in a myriad of neurosurgical patients which entails its tunneling. We describe a safe and simple technique of catheter insertion and tunneling using two Tuohy needles instead of one, which prevents unintentional catheter damage during the procedure.

An intrathecal lumbar drain placed for extended periods of time is warranted in conditions, such as perioperative drainage of cerebrospinal fluid (CSF) to facilitate brain relaxation in patients undergoing craniotomy or transsphenoidal surgery, access for intrathecal medication administration, during repair of thoracoabdominal aortic aneurysms, and as a CSF diversion in the treatment of cranial or spinal CSF leaks. ${ }^{1,2}$ To evade complications such as catheter migration, kinking, leaks, and infection, ${ }^{2-4}$ tunneling of the catheter is routinely practised. ${ }^{3}$ We report a safer technique of tunneling using two Tuohy needles instead of the prior described method involving one needle. ${ }^{3}$

A written informed consent was obtained from the patient for publishing images prior to writing this note.

In lateral decubitus position, lumbar puncture (LP) is performed at L3-4 or L4-5 interspace using a Tuohy needle $(14 \mathrm{G})$ under aseptic precautions. The lumbar catheter is advanced into the subarachnoid space through the needle subsequent to the removal of stylet and turning the bevel of the needle cephaloid to ensure advancement of the catheter in an upward direction. The needle and catheter unit is left in situ ( $\bullet$ Fig. 1A). The second Tuohy needle (14G) is now used to create a subcutaneous tunnel starting about $6 \mathrm{~cm}$ lateral to the LP entry site and brought out adjacent to the LP site (-Fig. 1B). A small stab incision $(2 \mathrm{~mm})$ is made at the LP site to facilitate this. Furthermore, one should ensure there is no skin tag between the insertion needle and the tunneling needle. Following removal of the first Tuohy needle carefully, the free end

Published online

Jun 24, 2020
DOI https://doi.org/

10.1055/s-0039-1692593

ISSN 2348-0548. of the catheter is fed through the second Tuohy needle (beveled end) using non-toothed forceps and brought out through the needle hub ( - Fig. 1C). The second Tuohy needle is then removed and the catheter is secured ( - Fig. 1D). An external drainage bag is connected to the free end of the catheter.

A simple procedure of lumbar drainage catheter insertion can be plagued by complications during tunneling when single needle is used for LP and tunneling as described previously by Hahn et al. ${ }^{3}$ Our method of lumbar drain tunneling is similar to their technique with a few exceptions. First, our method utilizes two Tuohy needles instead of one. Second, the crucial step of not removing the first Tuohy needle till the second one is tunneled prevents damage to the catheter, as tunneling is associated with a considerate amount of manipulation with an unpredictable give-way at the time of emergence of the needle tip. This two-needle technique circumvents the complication of catheter damage. Finally, a 2-mm stab incision at LP site suffices in our method as opposed to $5 \mathrm{~mm}$ described by them, which may require a skin suture.

The two-needle technique for tunneling of lumbar drain catheter has been routinely practiced by us close to two decades in over 500 cases. We have not encountered any complications so far attributable to the technique per se. We used a Tuohy needle $(14 \mathrm{G})$ available separately as a single needle with stylet for tunneling. However, an old LP needle may be reused after sterilization.

We recommend the two-needle method for tunneling of lumbar drain catheter to prevent inadvertent catheter 


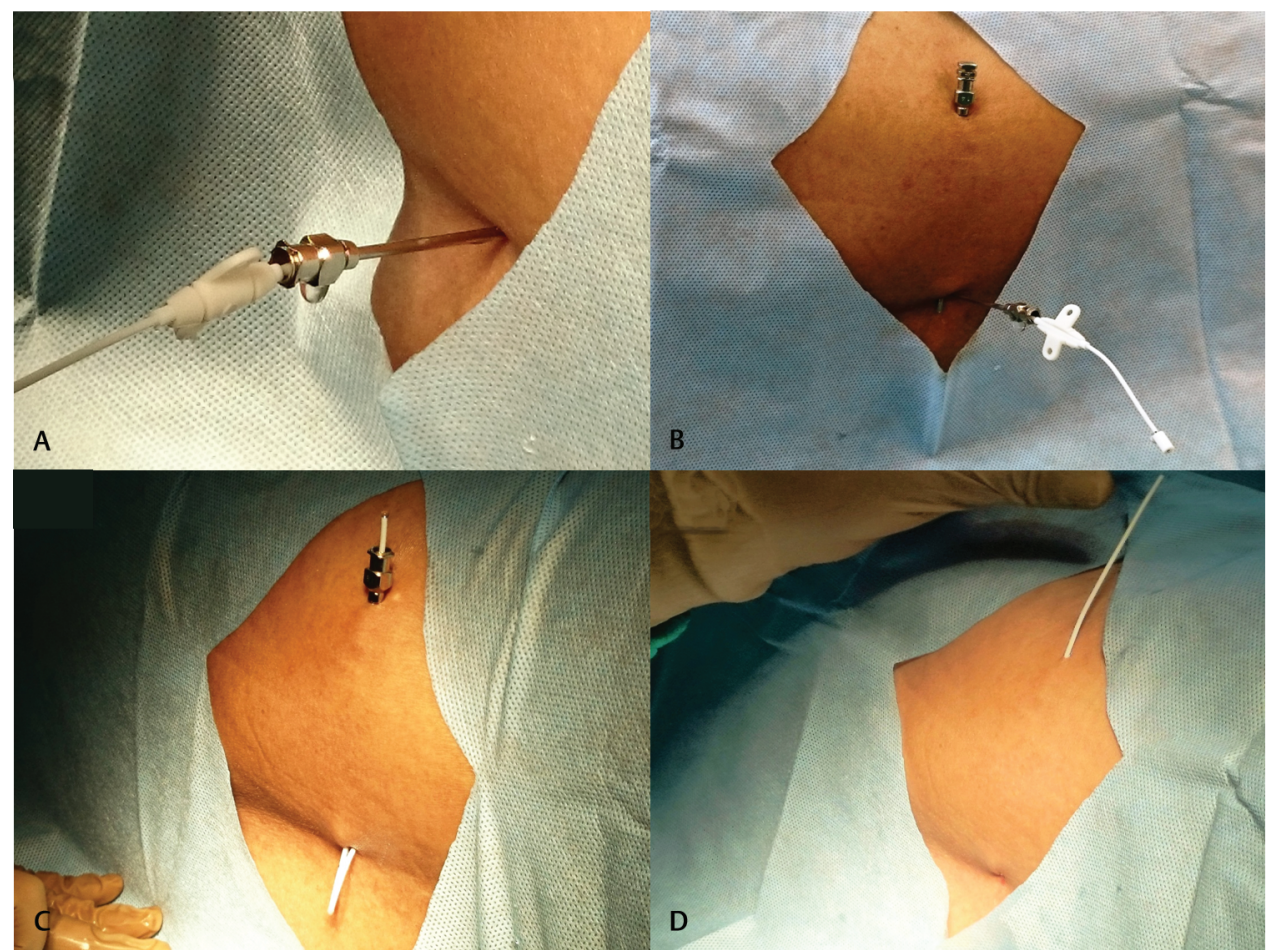

Fig. 1 (A) Lumbar drain catheter inserted into subarachnoid space through first Tuohy needle. (B) Subcutaneous tunnel created using second Tuohy needle leaving the first one in situ. (C) Free end of catheter brought out through the tunneled needle. (D) Final catheter position following removal of both needles.

damage while tunneling. The retained LP needle functions like a shield, while rest of the procedure is underway.

\section{Conflict of Interest}

None declared.

\section{References}

1 Hnenny L, Sabry HA, Raskin JS, Liu JJ, Roundy NE, Dogan A. Migrating lumbar intrathecal catheter fragment associated with intracranial subarachnoid hemorrhage. J Neurosurg Spine 2015;22(1):47-51

2 Shapiro SA, Scully T. Closed continuous drainage of cerebrospinal fluid via a lumbar subarachnoid catheter for treatment or prevention of cranial/spinal cerebrospinal fluid fistula. Neurosurgery 1992;30(2):241-245

3 Hahn M, Murali R, Couldwell WT. Tunneled lumbar drain. Technical note. J Neurosurg 2002;96(6):1130-1131

4 Follett KA, Naumann CP. A prospective study of catheterrelated complications of intrathecal drug delivery systems. J Pain Symptom Manage 2000;19(3):209-215 